\title{
IMAGE REGISTRATION USING ROBUST CORRELATION
}

\author{
Jeongtae Kim and Jeffrey A. Fessler \\ Electrical Engineering and Computer Science \\ University of Michigan, Ann Arbor \\ e-mail:jeongtae@umich.edu
}

\begin{abstract}
We have investigated an intensity-based image registration technique using a robust correlation coefficient as a similarity measure. The proposed method has an advantage over the ordinary correlation coefficient since it reduces the effect of "outlier" image intensity values. For the application of image registration to radiotherapy or image-guided surgery, there may be outlier samples due to the presence of the objects such as surgical instruments. We have verified the usefulness of the proposed method by simulation and phantom experiment.
\end{abstract}

\section{INTRODUCTION}

Image registration is a useful technique for diagnosis, patient set-up estimation for radiation therapy[1], tracking for image-guided surgery[2][3], etc. For set-up estimation and tracking problem, a pre-operative image is geometrically transformed to achieve the registration, and the patient setup is estimated as the geometrical transformation that accomplishes the registration. Intensity-based registration methods achieve registration by maximizing a similarity measure based on the intensity values of the two images. Therefore, designing an effective similarity measure is very important. The correlation coefficient between images is one of the most widely used similarity measures, and is appropriate when two images are from the same modality imaging devices [4][5]. The correlation coefficient is usually estimated by the sample correlation coefficient. Although the sample correlation coefficient has many distribution free advantages such as unbiasedness and consistency, the sample correlation coefficient is very sensitive to outliers[6]. A few outlier values can effect the sample correlation coefficient greatly. This can degrade performance in image registration. In fact, a significant number of "outliers" are present in the image-guided surgery application due to the presence of the operational instrument. For radiotherapy, outliers may be present due to the effect of radiotherapy table.

This work was supported by NIH Grant CA60711, P01-CA59827 and R01-CA81161
To remedy this problem, we have investigated a robust correlation coefficient as a similarity measure for image registration. We have used an affinely invariant approach[6] to calculate the robust correlation coefficient. To evaluate the performance of the proposed registration method, we conducted an experiment with an anthropomorphic chest phantom. We evaluated the performance of the proposed method by comparisons with the sample correlation coefficient method based on the "ground truth" determined by a marker-based method.

\section{ESTIMATING COVARIANCE MATRIX}

This section reviews the ordinary sample correlation coefficient and describes the proposed robust correlation coefficient.

\subsection{MLE(Maximum Likelihood Estimator)}

Suppose that corresponding image intensity value pairs $\left\{x_{i}\right\}$ are the samples of two dimensional random vector $X$ whose pdf is an elliptic density that is transformed by nondegenerate transformation $x \rightarrow V(x-t)$ from a spherically symmetric density $f(\|x\|)$ in $R^{2}$. Then, the elliptic density is defined as following,

$$
f(x ; t, V)=|\operatorname{det} V| f(\|V(x-t)\|) .
$$

Suppose that a random vector $X_{0}=V(X-t)$ has a pdf $f\left(\left\|x_{o}\right\|\right)$ and the covariance matrix $K_{0}=\sigma^{2} I$, where $I$ is 2 by 2 identity matrix. Then, the mean and the covariance matrix of the random variable $X$ is $t$ and $\sigma^{2}\left(V^{T} V\right)^{-1}$. Therefore, the MLE of the mean and the covariance of the random variable $X$ can be determined by maximizing the likelihood function from eq. (1). By taking derivative with respect to $t$ and $V$, the MLE is obtained by satisfying the following implicit equations[6],

$$
\begin{array}{r}
\operatorname{ave}\{w(\|y\|) y\}=0 \\
\operatorname{ave}\left\{w(\|y\|) y y^{T}-I\right\}=0
\end{array}
$$


where, "ave" denotes sample averages, and

$$
w(y) \triangleq-\frac{f^{\prime}(y)}{y f(y)} .
$$

We can rewrite above implicit equations as followings:

$$
\begin{aligned}
t & =\frac{\operatorname{ave}\{w(\|y\|) x\}}{\operatorname{ave}\{w(\|y\|)\}} \\
\left(V^{T} V\right)^{-1} & =\operatorname{ave}\left\{w(\|y\|)(x-t)(x-t)^{T}\right\} .
\end{aligned}
$$

If we solve above equations with assuming $f\left(\left\|x_{0}\right\|\right)$ as the normal distribution with identity covariance matrix, then $w=1$ and the implicit equations is solved explicitly. The MLE for the mean and the covariance are the sample mean and the sample covariance matrix. As is well known, it can be said that the sample correlation coefficient is the maximum likelihood estimation of correlation coefficient for normal density $f\left(\left\|x_{0}\right\|\right)$.

For any distribution, after solving the implicit equations for $t$ and $V$, one can compute the function $w$ with estimated $t$ and $V$. Since, it weighs each sample according to its location, it may be called weighting function.

\subsection{Robust Estimator}

The sample mean and the sample covariance estimation are sensitive to outliers. Roughly speaking, this can be explained because the sample mean and the covariance are the MLE for the normal distribution. Since normal distribution has "lighter tails" and the MLE considers outliers as true samples, the effect of outlier is large. Conversely, if an estimator is the MLE for a distribution which has "heavy tails", the outlier may be considered to belong tail area and result in smaller change in likelihood function. Therefore, we may design more robust estimator by assuming a distribution that has heavier tails such as Laplacian. For this paper, we have picked a distribution whose pdf is

$$
f(\|x\|)=c e^{-\sqrt{1+\|x\|^{2} / \delta^{2}}-1}
$$

where, $c$ is an appropriate constant.

For this distribution, $w(y)$ is defined as following;

$$
w(y)=\frac{1}{\delta^{2} \sqrt{1+\frac{y^{2}}{\delta^{2}}}} .
$$

We solve the implicit equations (5),(6) for estimating $t$ and $V$ iteratively by following the algorithm described in [6].

One can show that estimating the correlation coefficient is independent from the choice of $\delta$, although estimating $t$ and $V$ depends on $\delta$. This is convenient property since we can reduce one design parameter. Therefore, the only design parameter for robust estimator using our approach is the selection of the pdf shape in (7). The optimal shape will presumably depend on the nature of the "outliers", but any pdf with heavier-tails than a normal distribution will improve robustness relative to the sample correlation coefficient.

\section{SIMULATION RESULTS}

To evaluate the statistical properties of the proposed method, we have implemented a simulation. We simulated mixture of two two-dimensional normal distributions that have true distribution with zero mean, unity variancès, correlation coefficient 0.93 and the outlier distribution with mean -1.3 , 1.5 variances $1,0.001$ and correlation coefficient 0.01 . The number of samples from the true distribution is 100000 and from the outlier distribution is 5000 . We repeated the simulation for 1000 realizations. Fig.1 shows the 2D histogram of the samples and the weighting function after solving the implicit equations.

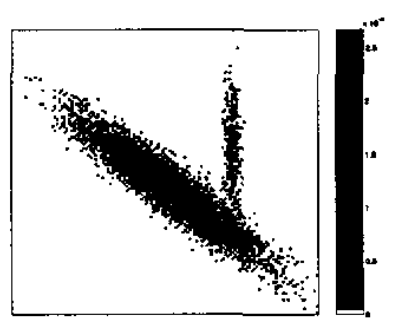

(a) Joint histogram

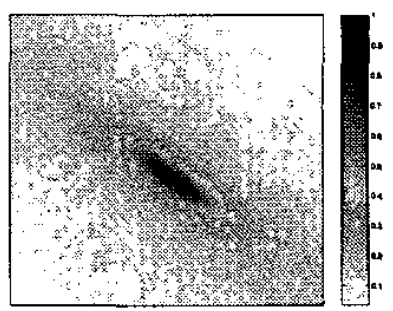

(b) Weighting function
Fig. 1. Distribution and weighting function.

Fig.2 shows the histograms of the estimated sample correlation coefficient, robust correlation coefficient and sample correlation coefficient without outlier samples. As we expected, the sample correlation coefficient is largely biased due to the outlier samples as well as the standard deviation is large. The robust estimator has reduced the bias and the variance greatly.

\section{EXPERIMENTAL RESULTS}

In our previous research[1], we conducted an anthropomorphic phantom experiment to evaluate the performance of the set-up estimators by $3 \mathrm{D} / 2 \mathrm{D}$ image registration. The estimation problem was estimating six parameters that are rotations and translations along the $\mathrm{X}, \mathrm{Y}, \mathrm{Z}$ axis using two orthogonal images. We used the same data set for this research but used only one lateral image and tried to esti- 


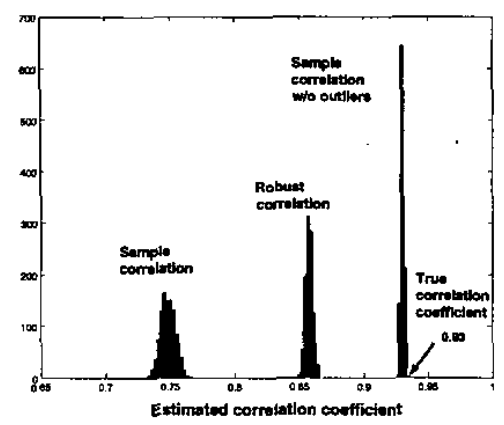

Fig. 2. Histograms of the estimators:sample correlation, robust correlation, sample correlation w/o outliers

mate one rotation parameter and two translation parameters that can be estimated using one image. The reason why we used only one image is that the lateral image only has outliers that are generated by the effect of the radiotherapy table. For this research, the other three parameters were kept fixed at the "ground truth" position that are established by the most accurate marker-based method. To establish "ground truth", we attached eleven 1mm diameter lead markers to the exterior surface of an anthropomorphic phantom. A $512 \times 512 \times 398$ voxel CT image with $0.9375 \times 0.9375 \times 1 \mathrm{~mm}$ spacing was acquired on a GE CT $/ \mathrm{i}$ scanner with a $140 \mathrm{kV} x$-ray source. Tattoos were drawn on the phantom where three alignment laser planes crossed the phantom to facilitate consistent set-up in a treatment room. Next, the phantom was moved to the treatment room and it was set up at the isocenter by manually aligning tattoos to three laser planes in the treatment room. Four radiographs were obtained from different angles by rotating the $\mathrm{x}$-ray source and Varian Portal Vision amorphous silicon active matrix flat panel image detector in $30^{\circ}$ increments. For $90^{\circ}$ view, we acquired 10 repeated radiographs without realignment for evaluating the effect of noise on the estimator. The $\mathrm{x}$-ray source voltage was $6 \mathrm{MV}$ and the detector size was $512 \times 384$ pixels with $0.78 \mathrm{~mm} \times 0.78 \mathrm{~mm}$ spacing. We used only radiograph from $90^{\circ}$ (i.e, lateral image) for the correlation-based methods. However, to enhance the accuracy of the "ground truth", we used all four radiographs for the fiducial marker-based method. For the correlationbased methods, the planning CT image was down-sampled by four along each axis to reduce computation time and memory usage. For image registration, while geometrically transforming the CT image, we computed DRR(Digitally Reconstructed Radiograph) of the transformed CT from the same angle as the radiograph acquired. Then, the registration is achieved by maximizing the similarity measure between such DRR and radiograph. We used only the central
$400 \times 300$ sub-image of the DRR and the radiograph to avoid the effect of the markers which are not usually used in clinical practice. We have established the geometry of the EPID imaging systems by determining radiation field edges using simple thresholding method[7]. Fig. 3 shows the radiograph and the DRR at the registered position. We can see the effect of the radiotherapy table around the rightmost parts of the radiograph. Pixels around the right most parts of the radiograph are brighter than those from the DRR. This is because the radiotherapy table increased the attenuation coefficient.

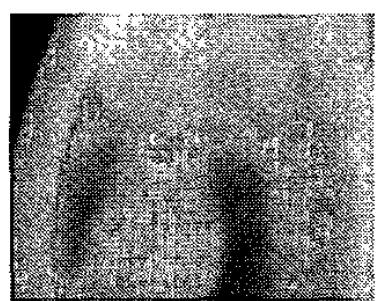

(a) Radiograph

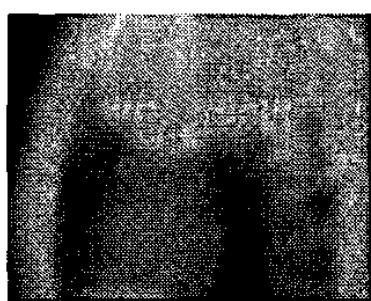

(b) DRR
Fig. 3. Radiograph and DRR.

Fig. 4 (a) shows the estimated joint histogram from the registered radiograph and the DRR. We can see some outlier distribution from mostly linear distribution. This is due to the presence of the radiotherapy table, as explained. The weighting function presented in Fig. 4 (b) reduces the effect of the outlier samples.

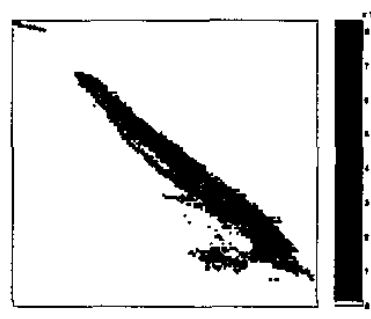

(a) Estimated pdf

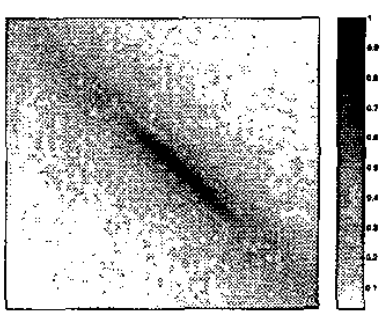

(b) Weighting function
Fig. 4. Estimated pdf and weighting factors

By applying the proposed robust correlation coefficient based method, we estimate one rotation and two translation parameter. Table 1 summarizes the experimental results. We repeated 10 estimations using 10 acquisitions of the radiograph. 
Table 1. Estimated set-up parameters

\begin{tabular}{|c||c||c||c|}
\hline & $\phi_{x}$ & $\bar{t}_{y}$ & $t_{z}$ \\
\hline Error (sample) & 1.39 & -2.06 & 2.11 \\
\hline Error (robust) & 1.07 & -0.99 & 1.33 \\
\hline \hline STD (sample) & 0.02 & 0.02 & 0.03 \\
\hline STD (robust) & 0.10 & 0.06 & 0.13 \\
\hline
\end{tabular}

(*The units for rotation parameter is degree and for translation parameters are $\mathrm{mm}$. STD is the standard deviation from 10 repetitions)

\section{DISCUSSION}

We have been able to reduce the errors of the sample correlation coefficient-based image registration using robust correlation coefficient as a similarity measure. We suspected that the error of the sample correlation coefficient-based registration was due to the outlier samples. By reducing the errors using robust method, we suspect that the fact was proved indirectly. However, for more rigorous arguments, we need to investigate that those errors are caused by the outliers in both mathematical way and experimental way. We also found that the STD of robust correlation coefficient based estimator was relatively larger than that of sample correlation coefficient based estimator in the phantom experiment. We have no knowledge whether it is for this particular experiment only or general. Addressing this problem is one of our future research goals. The other interesting research topic is the evaluation of the performance of the robust correlation coefficient to the other similarity measure such as MI(Mutual Information). We think that every estimator has trade-off between efficiency and robustness. Investigating that property among useful similarity measures can be valuable research topic. Finally, we can design many different robust correlation coefficient estimators by designing different pdf other than that used in this research. For example, Huber function type pdf can be an interesting estimator. Designing better estimator using some information such as the histogram of images may be valuable for the applications where the presence of the outliers are inevitable.

\section{CONCLUSION}

We have proposed a robust correlation coefficient as a similarity measure for the intensity-based image registration task. We have been able to reduce the bias of the sample correlation coefficient-based image registration by using the proposed method in a phantom experiment. We believed that the relatively large error of the sample correlation-based method was caused by the presence of outlier samples. We think that the robust correlation coefficient may be an effective similarity measure for the image registration task where the presence of the outlier is inevitable such as set-up estimation for radiotherapy and image-guided surgery.

\section{ACKNOWLEDGMENTS}

We are very grateful to James Balter, Kwok Lam and Randall Ten Haken from the Radiation Oncology Department in University of Michigan, Ann Arbor for providing the experimental data and bringing image registration problem for radiation therapy to us.

\section{REFERENCES}

[1] J Kim, J A Fessler, K L Lam, J M Balter, and R K Ten Haken, "A feasibility study of mutual information based set-up error estimator for radiotherapy," Medical Physics, vol. 28, no. 12, pp. 2391-2593, 2001.

[2] M J Murphy, "An automatic six-degree-of-freedom image registration algorithm for image-guided frameless stereotactic radiosurgery," Med. Phys., vol. 24, no. 6, pp. 857, June 1997.

[3] J Weese, G P Penney, P Desmedt, T M Buzug, D L G Hill, and D J Hawkes, "Voxel-based 2-d/3-d registration of fuoroscopy images and CT scans for image-guided surgery," IEEE Trans. on Info. Tech. in Biomedicine, vol. 1, no. 4, pp. 284-293, 1997.

[4] L Lemieux, R Jagoe, D R Fish, N D Kitchen, and D G T Thomas, "A patient-to-computed-tomography image registration method based on digitally reconstructed radiographs," Med. Phys., vol. 21, no. 11, pp. 1749-1760, 1994.

[5] L Dong and A L Boyer, "An image correlation procedure for digitally reconstructed radiographs and electronic portal images," Int J. Radiation Oncology Biol. Phys., vol. 33, no. 5, pp. 1053-1060, 1995.

[6] P J Huber, Robust Statistics, Wiley Series in Probability and Mathematical Statistics. John Wiley and Sons, 1981 .

[7] L Dong and A L Boyer, "A portal image alignment and patient setup verification procedure using moments and correlation techniques," Phys. Med. Biol., vol. 41, pp. 697-723, 1996. 